\title{
Impact of Swarm GPS receiver updates on POD performance
}

\author{
Jose van den IJssel ${ }^{1 *}$, Biagio Forte ${ }^{2}$ and Oliver Montenbruck ${ }^{3}$
}

\begin{abstract}
The Swarm satellites are equipped with state-of-the-art Global Positioning System (GPS) receivers, which are used for the precise geolocation of the magnetic and electric field instruments, as well as for the determination of the Earth's gravity field, the total electron content and low-frequency thermospheric neutral densities. The onboard GPS receivers deliver high-quality data with an almost continuous data rate. However, the receivers show a slightly degraded performance when flying over the geomagnetic poles and the geomagnetic equator, due to ionospheric scintillation. Furthermore, with only eight channels available for dual-frequency tracking, the amount of collected GPS tracking data is relatively low compared with various other missions. Therefore, several modifications have been implemented to the Swarm GPS receivers. To optimise the amount of collected GPS data, the GPS antenna elevation mask has slowly been reduced from $10^{\circ}$ to $2^{\circ}$. To improve the robustness against ionospheric scintillation, the bandwidths of the GPS receiver tracking loops have been widened. Because these modifications were first implemented on SwarmC, their impact can be assessed by a comparison with the close flying Swarm-A satellite. This shows that both modifications have a positive impact on the GPS receiver performance. The reduced elevation mask increases the amount of GPS tracking data by more than $3 \%$, while the updated tracking loops lead to around $1.3 \%$ more observations and a significant reduction in tracking losses due to severe equatorial scintillation. The additional observations at low elevation angles increase the average noise of the carrier phase observations, but nonetheless slightly improve the resulting reduced-dynamic and kinematic orbit accuracy as shown by independent satellite laser ranging (SLR) validation. The more robust tracking loops significantly reduce the large carrier phase observation errors at the geomagnetic poles and along the geomagnetic equator and do not degrade the observations at midlatitudes. SLR validation indicates that the updated tracking loops also improve the reduced-dynamic and kinematic orbit accuracy. It is expected that the Swarm gravity field recovery will benefit from the improved kinematic orbit quality and potentially also from the expected improvement of the kinematic baseline determination and the anticipated reduction in the systematic gravity field errors along the geomagnetic equator. Finally, other satellites that carry GPS receivers that encounter similar disturbances might also benefit from this analysis.
\end{abstract}

Keywords: Swarm, GPS, Precise orbit determination, lonospheric scintillation, Elevation mask, Tracking loop

\section{Background}

The European Space Agency (ESA) Swarm mission was launched on 22 November 2013 and consists of three identical satellites flying in carefully selected near polar orbits. Two satellites fly almost side-by-side at an initial altitude of about $480 \mathrm{~km}$ and will descend due to drag

\footnotetext{
*Correspondence: j.a.a.vandenijssel@tudelft.nl

${ }^{1}$ Faculty of Aerospace Engineering, Delft University of Technology, Delft, The Netherlands

Full list of author information is available at the end of the article
}

to around $300 \mathrm{~km}$ during the mission lifetime. The third satellite was placed in a higher orbit of about $530 \mathrm{~km}$ altitude and therefore descends much more slowly. The primary mission objective is to study the dynamics of the Earth's magnetic field and its interaction with the Earth system (Friis-Christensen et al. 2008). To achieve these science goals, the Swarm satellites are equipped with a number of instruments, such as scalar and vector magnetometers and an electrical field instrument. An accelerometer instrument is also part of the Swarm satellite payload and is used to derive information about the

\section{Springer Open}


thermospheric density and winds, which is a secondary Swarm mission objective (Visser et al. 2013).

In addition, each Swarm satellite is equipped with two state-of-the-art Global Positioning System (GPS) receivers, which are developed by RUAG space (Zangerl et al. 2014). The main GPS receiver is running in nominal operations, whereas the other one serves as redundant unit. The GPS receivers are used to precisely geotag the magnetic and electric field observations. In addition, they are used to calibrate and precisely geolocate the onboard accelerometer observations. Unfortunately, the Swarm accelerometers suffer from a variety of unexpected disturbances, which severely affects their usefulness at especially the lower frequencies. Therefore, a dedicated processing strategy has been developed to convert the accelerometer observations to thermospheric neutral densities, which relies on nongravitational accelerations that are derived from the precise Swarm GPS tracking data to determine the low-frequency information (Siemes et al. 2015). Swarm GPS high-low satellite-to-satellite tracking observations can also be used to determine the Earth's gravity field (Jäggi et al. 2015). Using Swarm GPS data for the continued monitoring of the Earth's time-variable gravity field could become especially relevant during a potential gap between the current GRACE mission and the planned GRACE Follow-On mission, which is scheduled for launch in 2017 (Flechtner et al. 2014). Finally, the Swarm GPS tracking data are used to estimate the slant total electron content, which is the integrated electron density along the line of sight from the Swarm satellite to the GPS satellites (Hoque et al. 2014).

The Swarm GPS receivers have eight channels available for dual-frequency tracking of GPS satellites and deliver high-quality GPS data with an almost continuous data availability. Initially, the Swarm GPS data were delivered with a $0.1 \mathrm{~Hz}$ data rate. Since 15 July 2014, however, the GPS data rate has been increased to $1 \mathrm{~Hz}$, to allow a near simultaneous sampling of the GPS observation epochs for the different Swarm satellites, which benefits the determination of baselines between the Swarm satellites. The Swarm GPS receivers have a hard-coded elevation mask, which was initially set to $10^{\circ}$. With this setting, the Swarm GPS receivers are able to track around $50 \%$ of the time the maximum number of eight satellites (van den IJssel et al. 2015). Precise science orbits (PSOs) for the Swarm satellite constellation are computed using the GPS tracking observations in the framework of the Swarm Satellite Constellation Application and Research Facility (Olsen et al. 2013). These PSOs nominally consist of both a reduced-dynamic and a kinematic orbit solution, which have an accuracy of, respectively, better than $2 \mathrm{~cm}$ and about $4-5 \mathrm{~cm}$, as shown by independent satellite laser ranging (SLR) validation (van den IJssel et al. 2015).
Despite this good performance, the Swarm GPS receivers show a slightly degraded performance when flying close to the geomagnetic poles and the geomagnetic equator. At these locations, the GPS carrier phase residuals resulting from the reduced-dynamic and kinematic orbit computations are systematically larger than in other parts of the orbit (van den IJssel et al. 2015; Jäggi et al. 2015). Because of the additional use of orbit dynamics, the reduced-dynamic orbits are less affected by this degraded GPS tracking data. The kinematic orbits, however, are more affected due to the stronger dependency of kinematic orbits on GPS data quality and show larger errors at these locations (van den IJssel et al. 2015; Jäggi et al. 2015). The degraded performance of the Swarm GPS receivers over these locations also affects the kinematic baseline determination between the lower flying pair. Jäggi et al. (2015) show that Swarm kinematic baselines have significantly larger errors over the geomagnetic poles, while Allende-Alba and Montenbruck (2015) show that when these areas are excluded, the rms error of the Swarm kinematic baselines drops from the few $\mathrm{cm}$ to the few $\mathrm{mm}$ level. In addition, gravity fields determined from Swarm GPS data show severe systematic errors along the geomagnetic equator (Jäggi et al. 2015).

The particular locations where the Swarm GPS receivers show a degraded performance suggest that the receivers are affected by ionospheric scintillation. Ionospheric scintillation occurs when electromagnetic signals propagate through an irregular ionosphere. GPS signals are vulnerable to ionospheric scintillation, which can degrade or interrupt GPS receiver operations (Kintner et al. 2007). This is particularly true for semi-codeless tracking of the GPS $\mathrm{P}(\mathrm{Y})$-code signal due to the losses implied by this tracking technique (Woo 2000). The tracking performance of a GPS receiver under ionospheric scintillation conditions depends not only on the magnitude of the observed scintillation activity, but also on the receiver tracking capabilities (Skone et al. 2001). Differences in receiver performance are due to many factors, such as antenna gain patterns, internal processing algorithms and tracking loop bandwidths (Skone et al. 2001). For receivers in low-Earth orbit (LEO), an adverse impact of scintillation in polar regions and two belts around $15^{\circ}$ north and south of the geomagnetic equator has, for example, been reported in van den IJssel et al. (2011) and Kramer and Goodman (2005).

In order to improve the Swarm GPS receiver tracking performance, several modifications have been implemented to the onboard GPS receivers after launch. Table 1 gives an overview of these modifications and indicates when they were uplinked to the different satellites. Because the number of tracked GPS satellites is relatively low for the Swarm GPS receivers, compared to 
Table 1 Overview of Swarm GPS receiver modifications

\begin{tabular}{|c|c|c|}
\hline Date & Satellite & GPS receiver modification \\
\hline $21-10-14$ & Swarm-A & Antenna FoV from $80^{\circ}$ to $83^{\circ}$ \\
\hline \multirow[t]{2}{*}{$22-10-14$} & Swarm-B & Antenna FoV from $80^{\circ}$ to $83^{\circ}$ \\
\hline & Swarm-C & Antenna FoV from $80^{\circ}$ to $83^{\circ}$ \\
\hline $01-12-14$ & Swarm-C & Antenna FoV from $83^{\circ}$ to $86^{\circ}$ \\
\hline $13-01-15$ & Swarm-C & Antenna FoV from $86^{\circ}$ to $88^{\circ}$ \\
\hline \multirow[t]{3}{*}{$06-05-15$} & Swarm-A & Antenna FoV from $83^{\circ}$ to $88^{\circ}$ \\
\hline & Swarm-B & Antenna FoV from $83^{\circ}$ to $88^{\circ}$ \\
\hline & Swarm-C & Tracking loop modifications \\
\hline $08-10-15$ & Swarm-A & Tracking loop modifications \\
\hline $10-10-15$ & Swarm-B & Tracking loop modifications \\
\hline
\end{tabular}

receivers on other scientific LEO satellites like CHAMP, GRACE and GOCE, which generally have 12 channels available for dual-frequency tracking (Montenbruck et al. 2006), the GPS antenna elevation mask has slowly been reduced from $10^{\circ}$ to $2^{\circ}$, in the hope that the GPS tracking performance would increase. With the current GPS constellation, satellites in a low-Earth orbit usually have much more than eight GPS satellites in view, which means that for Swarm the number of tracked satellites is limited by the number of tracking channels. With a $10^{\circ}$ antenna elevation mask, however, the Swarm GPS receiver is tracking the maximum of eight GPS satellites only about $50 \%$ of the time, which suggests there is room for improvement.

In addition, to make the Swarm GPS receivers more robust for ionospheric scintillation, the receiver tracking loops have been adjusted. Small-scale irregularities in the electron density along the GPS radio signal propagation path cause ionospheric scintillation that appears as rapid fluctuations in the intensity and phase of the received signal (Skone et al. 2001). The rapid phase fluctuations can cause a Doppler shift in the GPS signal, which can exceed the bandwidth of the carrier phase tracking loop of the receiver. For the Swarm receivers, the bandwidth of the L1 carrier loop has therefore been increased by $50 \%$ from 10 to $15 \mathrm{~Hz}$, while the L2 carrier loop bandwidth was increased by $100 \%$ from 0.25 to $0.5 \mathrm{~Hz}$. The bandwidth of the $\mathrm{P}(\mathrm{Y})$ code loop was also increased by $100 \%$. In addition, the fast reacquisition of the L1-carrier tracking was enabled by increasing the retry counter from zero to five and the number of tracked GPS satellites required by the onboard Kalman filter for calculating a valid navigation solution was reduced from three to one (ESA 2015).

This paper presents an analysis of the impact of the different Swarm GPS receiver modifications on the GPS tracking performance and the resulting orbit quality. Because most of these modifications were first implemented on Swarm-C, their impact can be assessed by a comparison with Swarm-A. Swarm-A and SwarmC fly almost side-by-side in close formation, with a maximum separation between the two satellites of about $100 \mathrm{~km}$ in cross-track direction at the equator crossings, and therefore they are expected to have a similar GPS tracking performance when their receiver settings are identical. The precise orbit determination (POD) results given in van den IJssel et al. (2015) based on Swarm GPS data from the first 11 months of the mission indeed show a similar performance for the two satellites. However, these results include data obtained during the commissioning phase of the mission, when Swarm-A and Swarm-C were not yet flying in close formation, as well as data from December 2014, when both GPS receivers already had different elevation masks. Therefore, another comparison has been performed, using only data from 19 August to 14 October 2014, when the two satellites are in close formation with identical GPS receiver settings, and no large GPS data gaps or satellite manoeuvres occurred. The reduced-dynamic orbits computed for these 57 days show an average rms of the carrier phase residuals of 1.01 and $1.00 \mathrm{~cm}$ for, respectively, Swarm-A and Swarm-C and SLR residuals of $-0.08 \pm 2.00 \mathrm{~cm}$ for Swarm-A and $-0.26 \pm 2.06 \mathrm{~cm}$ for Swarm-C, which confirms the similar tracking performance of the two GPS receivers.

This paper first shows the analysis of the impact of the different GPS antenna elevation mask settings. Because the performance of the Swarm GPS receivers is affected by ionospheric scintillation, the next section provides background information about ionospheric scintillation effects on the Swarm GPS observations. In the following section, it is assessed whether the implemented tracking loop modifications improve the robustness of GPS tracking performance and the impact on both the reduceddynamic and kinematic orbit quality is shown. Finally, the paper concludes with a short summary of the results and gives recommendations for further improvements.

\section{Swarm GPS antenna elevation mask modifications}

At the start of the mission, the field of view (FoV) of the Swarm GPS antennas was set at a nominal value of $80^{\circ}$. This limit implies that only satellites within a cone of less than $80^{\circ}$ about the zenith direction are considered for tracking. In forward direction, satellites can first be tracked when their elevation rises beyond $10^{\circ}$, even though a small delay will be experienced before good and valid data are acquired. Once allocated and tracked, a satellite will remain allocated to a given channel until it sets below $10^{\circ}$ in the aft-looking hemisphere. Due to the limited amount of channels as compared to the number of visible satellites, it is not always possible to track newly visible satellites right from the beginning. Some satellites 
may only be allocated once they have already reached a high elevation.

In an attempt to increase the amount of GPS tracking observations, the antenna FoV has been adjusted several times during the mission. An overview of these changes is given in Table 1. This table shows that the $86^{\circ}$ and $88^{\circ}$ FoV settings were first implemented on Swarm-C only. The impact of these settings with respect to the $83^{\circ}$ setting can therefore be assessed by comparing the lower flying satellite pair. Based on this comparison, the FoV of the GPS antennas on Swarm-A and Swarm-B has also been increased to $88^{\circ}$ on 6 May 2015 .

\section{Impact on collected GPS data}

Figure 1 shows for all Swarm satellites the daily averages of the number of tracked GPS satellites per epoch. This number is limited by the eight available dual-frequency tracking channels of the Swarm GPS receivers. During the first few months of the mission, on average between 7.3 and 7.4 GPS satellites are tracked per epoch. Changing the GPS antenna FoV in steps from $80^{\circ}$ up to $88^{\circ}$ clearly increases the amount of tracking observations. With a $88^{\circ}$ FoV setting, the Swarm satellites track on average about 7.6 GPS satellites per epoch. Compared with the nominal $80^{\circ} \mathrm{FoV}$ at the start of the mission, this is an increase in GPS tracking observations of more than $3 \%$.

Figure 2 gives an overview of the daily minimum elevation angles of the GPS observations in the antenna-fixed frame for all Swarm satellites, in both flight direction and aft direction. The flight and aft directions in the antennafixed frame have azimuth angles of, respectively, $0^{\circ}$ and $180^{\circ}$. To obtain the minimum elevation angles in flight direction shown in Fig. 2, all observations with azimuth angles between $350^{\circ}$ and $10^{\circ}$ are used, while the aft direction contains all observations between $170^{\circ}$ and $190^{\circ}$.
There are several distinct changes in these minimum elevation angles, which are related to changes in the GPS receiver settings. Changing the GPS data rate from 0.1 to $1 \mathrm{~Hz}$ slightly decreases the minimum elevation angle for all satellites in flight direction. As expected, increasing the FoV decreases the minimum elevation angles. Figure 2 also shows a clear asymmetry between the flight and aft direction. In flight direction, the minimum elevation angle is a few degrees larger than expected from the FoV setting, while in aft direction, this angle is a few degrees lower. Ongoing investigations have not yet provided a convincing explanation for this asymmetry ( $C$. Siemes, personal communication).

The top panel of Fig. 3 shows for the lower flying Swarm satellite pair the distribution of the GPS observations in the antenna-fixed frame for the time period from 15 December 2014 to 12 January 2015. During these 29 days, the Swarm-A GPS antenna FoV was kept at $83^{\circ}$, while the Swarm-C FoV was increased to $86^{\circ}$. The distribution is typical for near polar satellites in a low-Earth orbit with a zenith-pointing GPS antenna. The vertical stripes are related to the orientation of the Swarm orbit relative to the individual orbital planes of the GPS constellation and the different number of GPS satellites populating each plane. GPS observations that are rejected in the POD computations are not included in this figure. During this period, Swarm-C collects around $1.04 \%$ more observations compared with Swarm-A. After data editing, however, this number slightly reduces to around $0.96 \%$. For Swarm-A, $37.55 \%$ of the GPS observations are collected in the forward direction of the antenna and $62.45 \%$ in the aft direction. For Swarm-C, $36.46 \%$ of the observations are in forward direction and $63.54 \%$ in aft direction. Most of the additional observations due to the increased FoV can be found in the aft direction of the GPS antenna, which means that the forward-backward

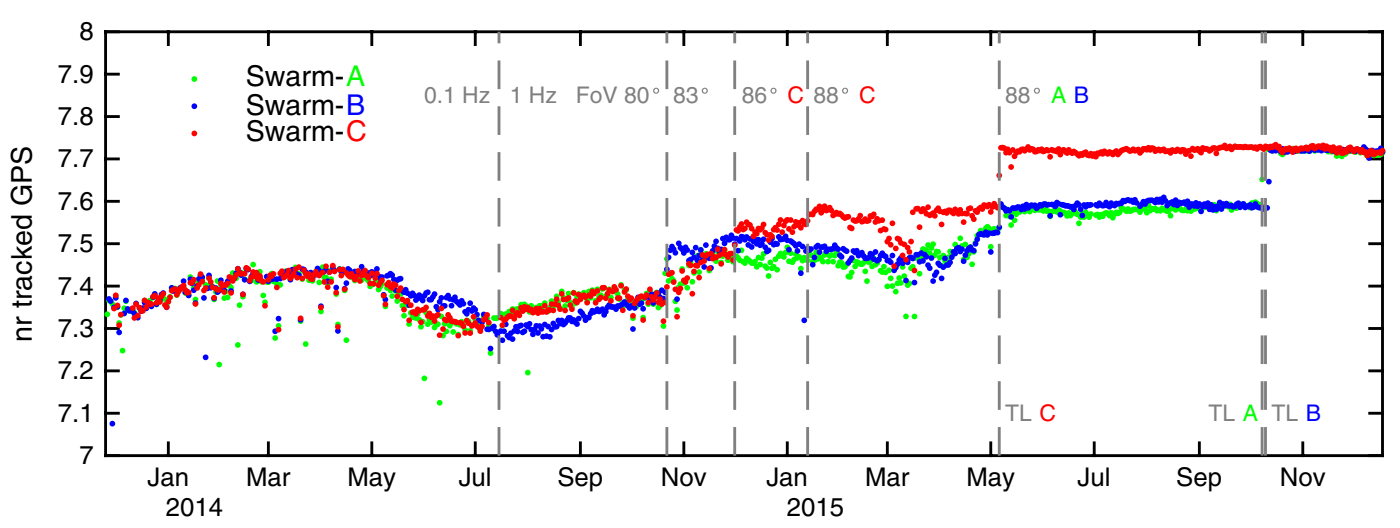

Fig. 1 Daily average number of tracked GPS satellites per epoch for each Swarm satellite. The grey dashed lines indicate GPS receiver modifications 

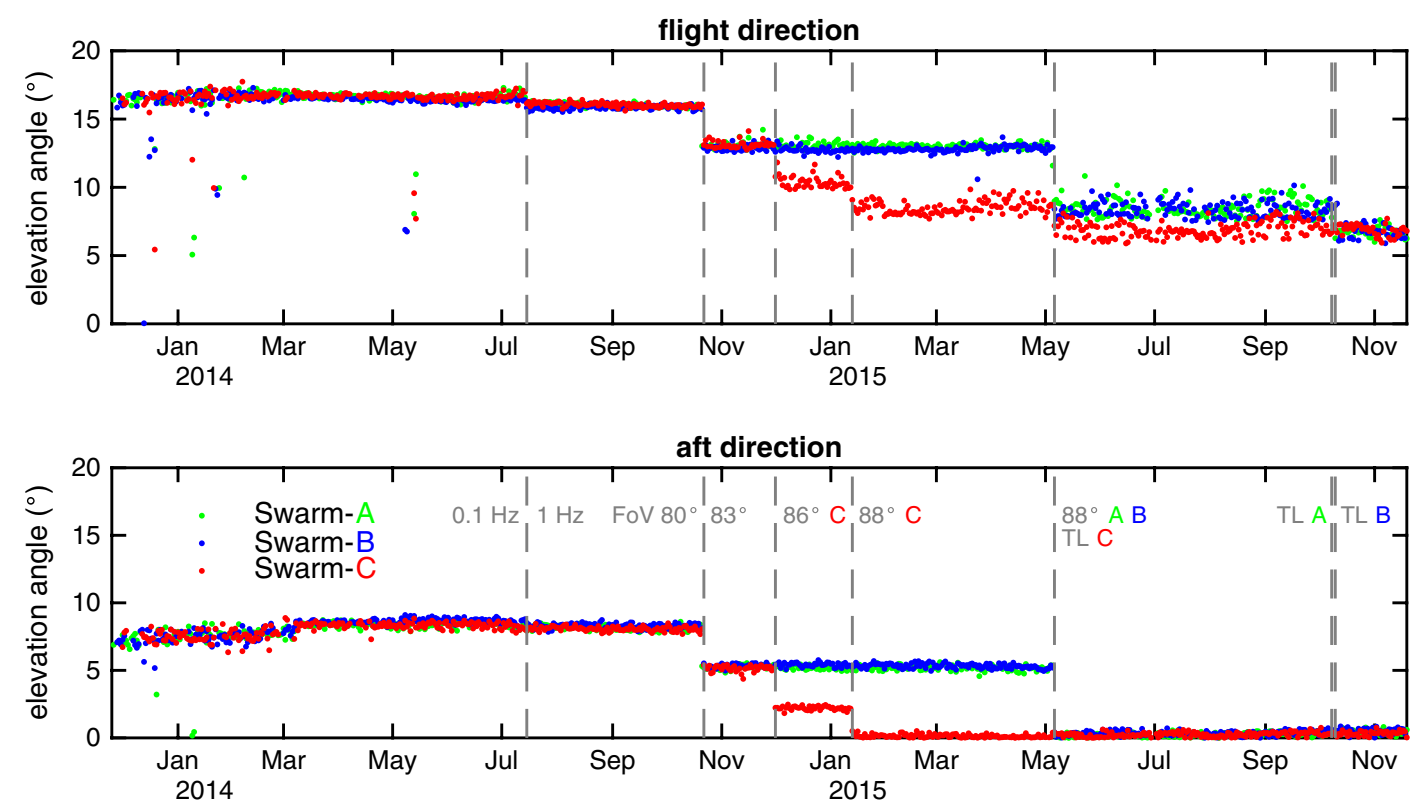

Fig. 2 Daily minimum elevation angles in the GPS antenna frame in flight (top panel) and aft (bottom panel) direction for each Swarm satellite. The grey dashed lines indicate GPS receiver modifications

asymmetry of the GPS observations in the antenna-fixed frame increases. This asymmetry might lead to a potential degradation of the geometric dilution of precision (DOP) of the GPS tracking observations in along-track direction, which could affect the quality of especially the kinematic orbit solutions.

The bottom panel of Fig. 3 shows the GPS observation distribution of Swarm-A and Swarm-C for the time period from 26 January to 3 March 2015. During these 37 days, the Swarm-A GPS antenna FoV remained at $83^{\circ}$, while the Swarm-C FoV was set to $88^{\circ}$. Swarm-C collects around $1.43 \%$ more GPS observations compared with Swarm-A. After data editing, this number reduces to around $1.15 \%$, which means that relatively more observations are rejected. Nonetheless, also after data screening relatively more GPS observations are available with the $88^{\circ} \mathrm{FoV}$ angle compared with $86^{\circ} \mathrm{FoV}$ angle. For SwarmA, $60.77 \%$ of the GPS observations are collected in the aft direction of the antenna, compared with $63.23 \%$ of the Swarm-C observations, which means that most of the additional observations can again be found in the aft direction of the GPS antenna.

\section{Impact on POD performance}

The processing strategy that is used to determine PSOs for the Swarm satellite constellation is explained in detail in van den IJssel et al. (2015). The reduced-dynamic and kinematic Swarm PSOs are computed using the GPS High-precision Orbit determination Software Tools
(GHOST), which are developed at the Deutsches Zentrum für Luft- und Raumfahrt in close cooperation with TU Delft (Wermuth et al. 2010). The POD strategy for both orbit types is based on an undifferenced approach and uses the ionosphere-free combination of GPS observations along with precise GPS orbits and high-rate 5-s clocks computed by the Center for Orbit Determination in Europe (CODE) (Bock et al. 2009). To determine the Swarm orbits with highest accuracy, it is necessary to take the GPS antenna phase center variations (PCVs) into account in the POD (van den IJssel et al. 2015; Jäggi et al. 2015). For low flying satellites like Swarm, these maps generally have to be determined during flight, because ground calibration does not properly account for the influence of error sources which are additionally encountered in the actual space environment, like near-field multipath.

Increasing the GPS antenna FoV requires a new computation of these PCV maps, in order to take the additional observations at low elevation angles into account. Figure 4 shows the recomputed PCV maps for all Swarm satellites. Similar to the original PCV maps, these maps are computed using the so-called residual approach, in which the estimated PCVs are derived as binwise mean values of the ionosphere-free GPS carrier phase residuals (Jäggi et al. 2009). Each map is based on 100 days of $1 \mathrm{~Hz}$ GPS data and includes 30 days with a GPS antenna $\mathrm{FoV}$ of $88^{\circ}$. In total, eight iterations have been performed, after which no further significant changes were visible 


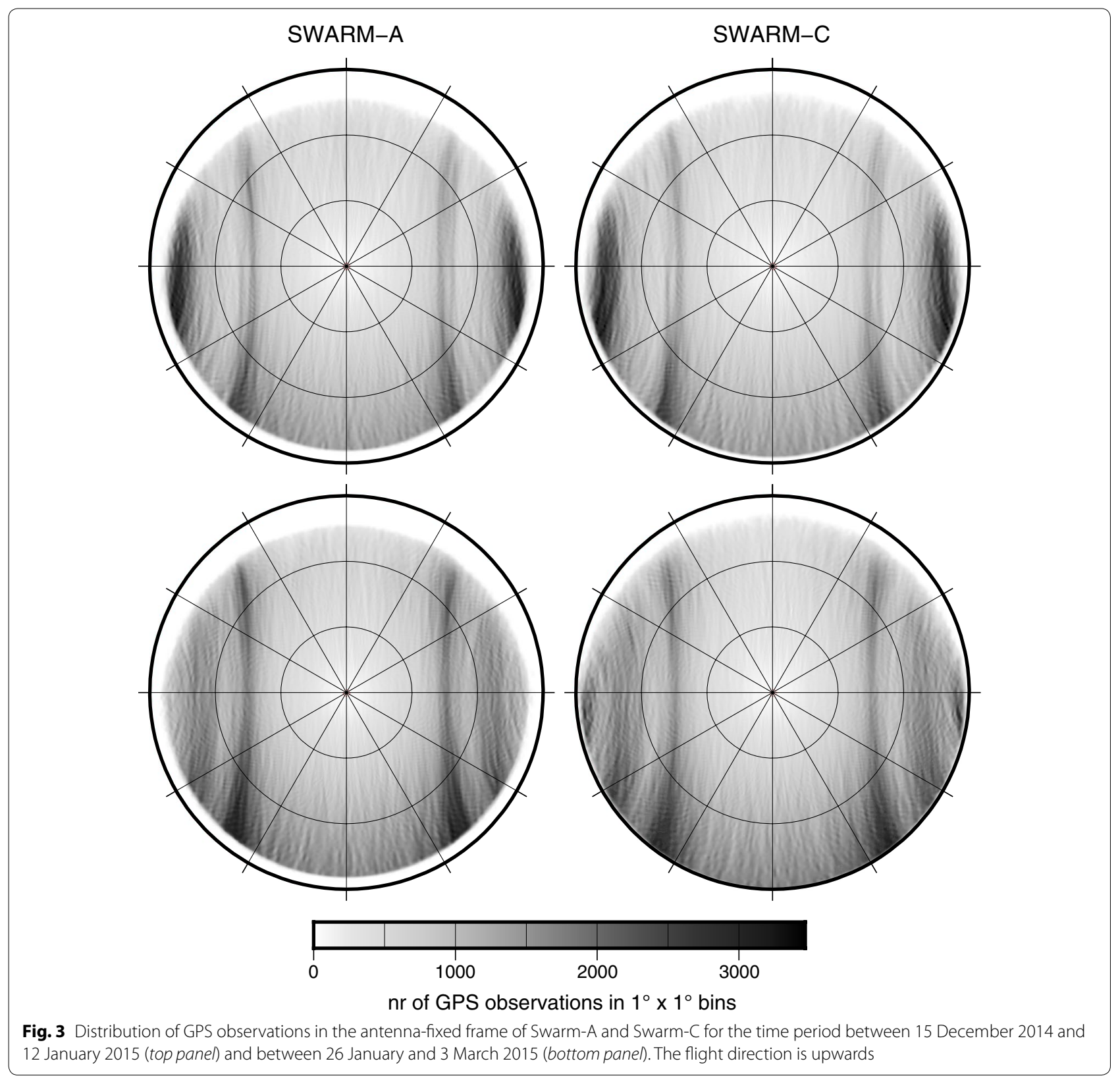

in the PCV maps and the resulting orbits. Except for the lowest elevation angles, these recomputed PCV maps are very similar to the PCV maps that were computed earlier, based on GPS data with a maximum FoV of $83^{\circ}$ for Swarm-A and Swarm-B or $86^{\circ}$ for Swarm-C (van den IJssel et al. 2015). At the additional low elevations, the estimated PCVs are relatively large, which shows that it is important to take them into account in the POD. Figure 4 also confirms the asymmetry of the antenna elevation mask that is already indicated in Figs. 2 and 3.

The impact of increasing the GPS antenna FoV from $83^{\circ}$ to $86^{\circ}$ or $88^{\circ}$ on the POD performance is assessed by a comparison between Swarm-A and Swarm-C using the same time periods that were used to assess the impact on the GPS observation distribution shown in Fig. 3. For both satellites, precise orbits have been computed using the nominal settings as described in van den IJssel et al. (2015), except that for Swarm-C the recomputed PCV map shown in Fig. 4 is used. Daily rms errors of the resulting ionosphere-free GPS carrier phase residuals are shown in the top panels of Figs. 5 and 6, which present, respectively, the comparison between the $83^{\circ}$ and $86^{\circ}$ FoV angles and the $83^{\circ}$ and $88^{\circ} \mathrm{FoV}$ angles. Both figures show that the ionosphere-free carrier phase residuals 


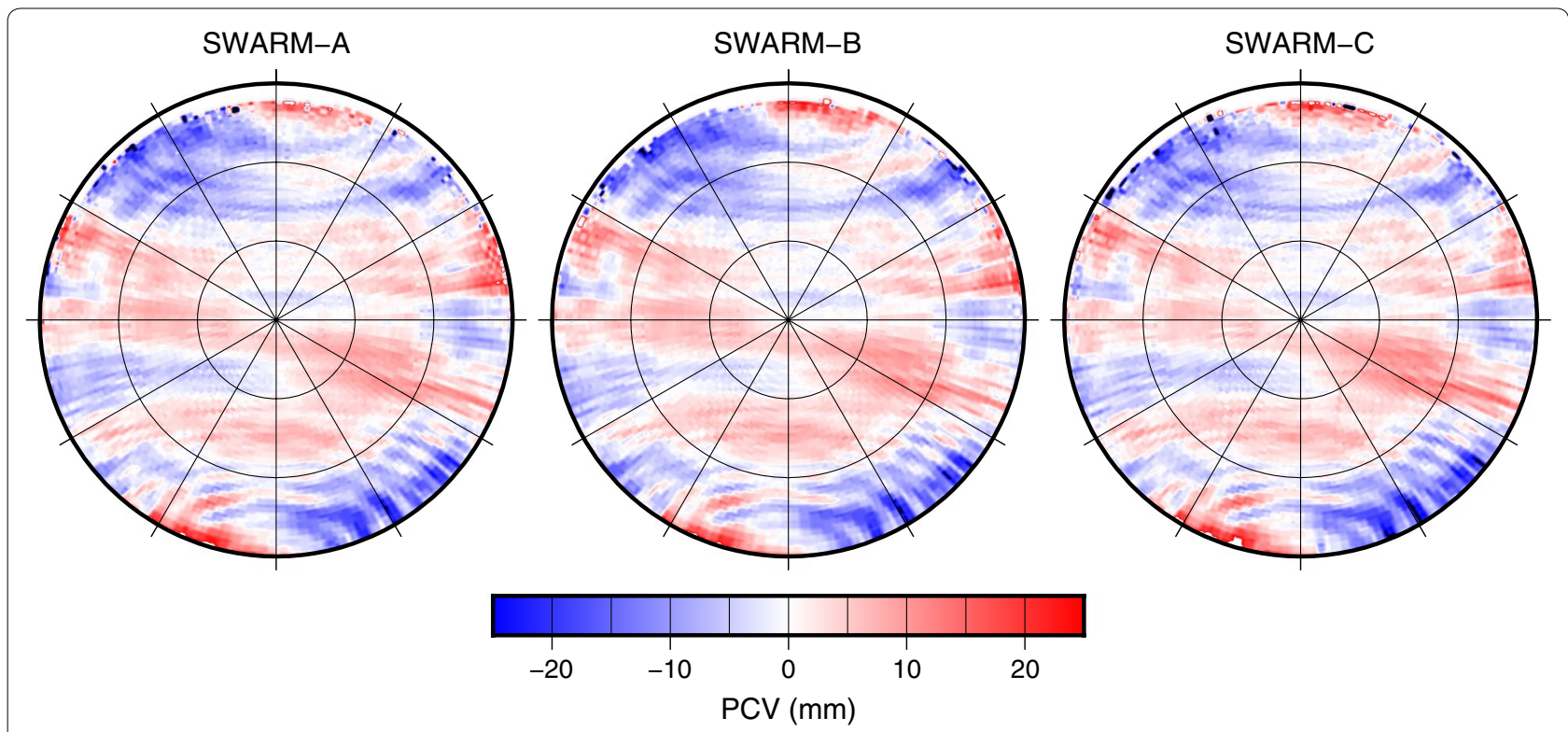

Fig. 4 Azimuth-elevation diagrams of estimated PCVs for the nominal Swarm POD antennas based on GPS data with a maximum FoV of $88^{\circ}$. The flight direction is upwards

for the reduced-dynamic and the kinematic orbits are slightly higher for Swarm-C compared with Swarm-A. This is due to the fact that the increased FoV of Swarm-C leads to additional observations at especially the low elevations, which are generally noisier. Compared with the results of Fig. 5, the differences between the two satellites shown in Fig. 6 are even larger, due to the increased amount of more noisy low elevation GPS observations for Swarm-C.

Because all Swarm satellites carry a laser retroreflector, independent SLR observations can be used to quantify the Swarm orbit accuracy. The SLR validation of the Swarm orbits is performed using observations from 18 high-quality ground stations of the International Laser Ranging Service tracking network (Pearlman et al. 2002). The processing strategy that is used to compute the SLR residuals is described in van den IJssel et al. (2015). To reject outliers which may still be present in the SLR data, a screening threshold of $25 \mathrm{~cm}$ was applied to the SLR residuals. The bottom panels of Figs. 5 and 6 show that the SLR residuals of Swarm-C for both orbit types are lower. This indicates that although the additional observations at elevations up to $86^{\circ}$ or $88^{\circ}$ are noisier, including them in the POD improves the orbit accuracy for both orbit types. Unfortunately, from this analysis it is not clear whether a $86^{\circ}$ or $88^{\circ} \mathrm{FoV}$ angle is optimal. Because slightly more GPS tracking data are obtained with the $88^{\circ} \mathrm{FoV}$ angle, this angle has meanwhile also been applied to the GPS antennas of Swarm-A and Swarm-B.

\section{lonospheric scintillation effects on Swarm GPS observations}

The zenith-pointing GPS receivers onboard of Swarm are flying at an altitude of about $500 \mathrm{~km}$, and therefore it was initially expected that ionospheric scintillation would not be a problem for these receivers (Zangerl et al. 2014). Contrary to these expectations, it was observed shortly after launch that the Swarm GPS observations are affected by ionospheric scintillation (Zangerl et al. 2014; Buchert et al. 2015). Although not impacting the primary science objectives of the mission, the scintillation-induced signal perturbations affect the quality of the kinematic POD, as well as the gravity field recovery (van den IJssel et al. 2015; Jäggi et al. 2015).

Ionospheric scintillation is the manifestation of the radio wave energy scattering through inhomogeneities in the spatial distribution of the refractive index. In the ionosphere, such inhomogeneities are associated with irregularities in the spatial distribution of the electron (or plasma) density, drifting across the ray path. Because of the relative motion between raypath, receiver, transmitter, and inhomogeneities, such a scattering manifests as temporal fluctuations in the intensity and phase of the received signal. Ionospheric scintillation is typically measured by means of statistical quantities. The amount of fluctuations appearing on the intensity of the received signal (i.e. intensity scintillation) is measured by means of the $S_{4}$ index, which is the standard deviation of the received intensity normalised by its average (Briggs and Parkin 1963) 

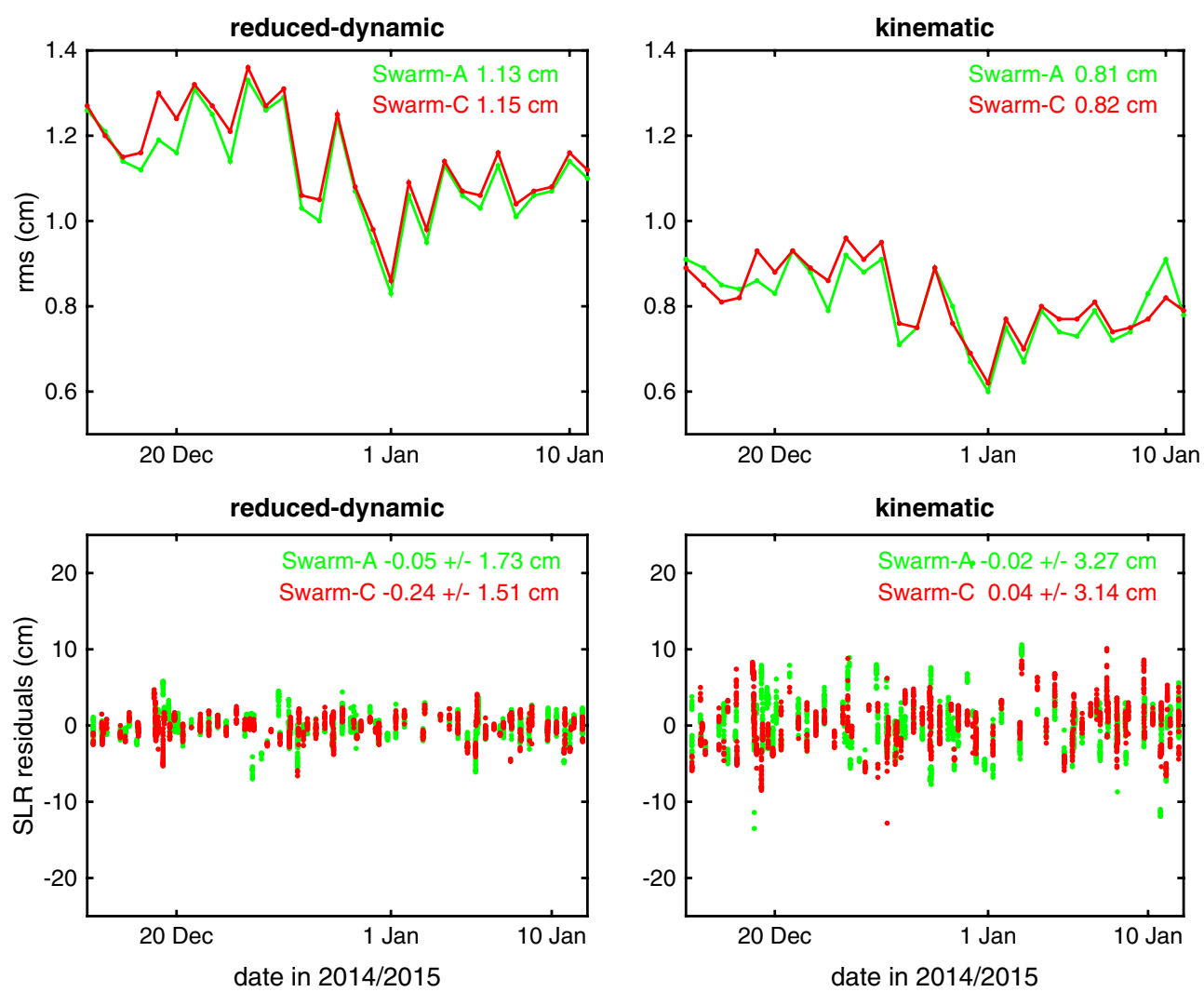

Fig. 5 Daily rms errors of the ionosphere-free carrier phase residuals for the reduced-dynamic orbits (top left panel) and kinematic orbits (top right panel), together with SLR residuals for the reduced-dynamic orbits (bottom left panel) and kinematic orbits (bottom right panel) of Swarm-A (83 FoV) and Swarm-C (86 FoV)

$$
S_{4}^{2}=\frac{\left\langle I^{2}\right\rangle-\langle I\rangle^{2}}{\langle I\rangle^{2}}
$$

where $I$ is the received intensity and $\langle\cdot\rangle$ denotes ensemble averaging. In real measurements, the typical assumption is that the observed scintillation phenomena are ergodic, and ensemble averaging is replaced by temporal averaging. The amount of fluctuation appearing on the carrier phase of the received signal (i.e. phase scintillation) is measured by means of the standard deviation of the received carrier phase $\sigma_{\phi}$, which has to be de-trended in order to remove the effect of the satellite motion. In ground-based Global Navigation Satellite System (GNSS) receivers, this de-trending is usually obtained by highpass filtering the carrier phase with a cut-off frequency at $0.1 \mathrm{~Hz}$ (van Dierendonck et al. 1993).

The occurrence and intensity of ionospheric scintillation depends on e.g. geographical location, local time, season, solar cycle and geomagnetic activity. The plasma inhomogeneities that are capable of producing radio wave scintillation at $\mathrm{L}$ band maximise around the equatorial anomaly and, to a lesser extent, at auroral and polar latitudes (Aarons 1982; Basu et al. 1988). Around the equatorial anomaly, these plasma inhomogeneities predominantly occur in the hours after sunset, while at high latitudes they can occur at any local time. The seasonal pattern shows minimum scintillation during summer and maxima during the equinoxes (Basu et al. 1988; Kintner et al. 2007). This is confirmed in Fig. 7, which shows monthly maps of the ionospheric variability detected by Swarm-A. These maps are derived from the geometry-free combination of the dual-frequency Swarm-A carrier phase observations, which is a measure of the ionospheric path delay on the L1 frequency derived from the dual-frequency carrier phases. For each map, the time derivatives of the geometry-free combination are accumulated over the respective month and averaged over $1^{\circ} \times 1^{\circ}$ bins.

For typical ground-based GNSS receivers, plasma inhomogeneities at low latitudes are capable of originating scintillation on both the intensity and phase of the received signal. At these latitudes, both weak scattering $\left(S_{4}<0.2\right)$ and strong scattering (where $S_{4}$ has higher values and can even saturate around 1) can occur, as a 

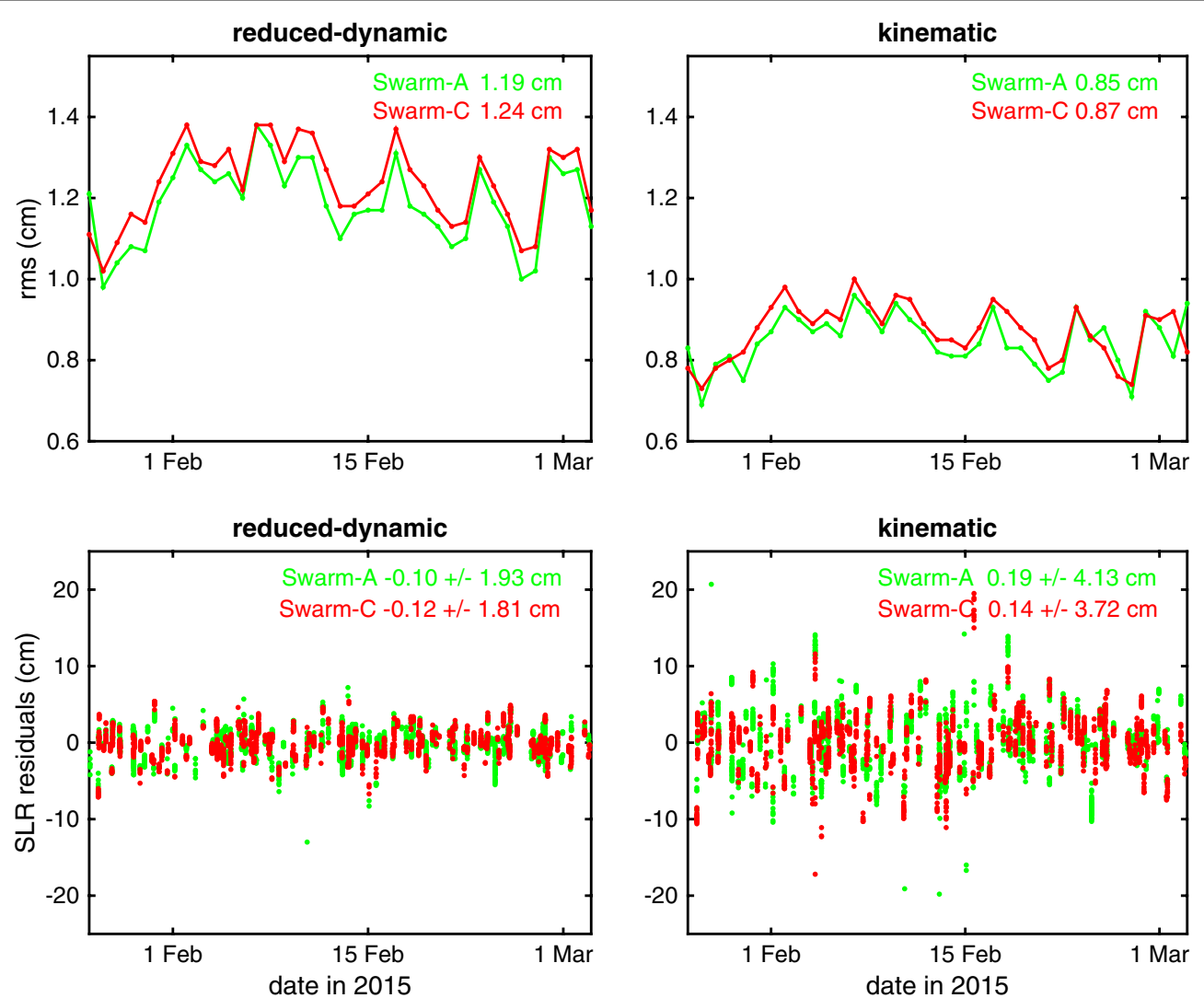

Fig. 6 Daily rms errors of the ionosphere-free carrier phase residuals for the reduced-dynamic orbits (top left panel) and kinematic orbits (top right panel), together with SLR residuals for the reduced-dynamic orbits (bottom left panel) and kinematic orbits (bottom right panel) of Swarm-A (83 ${ }^{\circ}$ FoV) and Swarm-C ( $\left.88^{\circ} \mathrm{FoV}\right)$

consequence of propagation through structures such as plasma bubbles and plumes of ionisation, which can extend to about $1000 \mathrm{~km}$ altitude. For GNSS receivers, intensity scintillation can cause fading which might lead to losses of lock on both L1 and L2. Phase scintillation reduces the accuracy of the carrier phase observables, as enhanced tracking errors propagate to the observables.

At auroral and polar latitudes, phase scintillation occurs rather than intensity scintillation, for example in the presence of particle precipitation, which induces ionisation between the $\mathrm{E}$ and $\mathrm{F}$ regions, and polar cap patches, which are typically propagating anti-sunward and extend to about $600 \mathrm{~km}$ in altitude. Due to the fieldaligned nature of these structures and because of the fast plasma drift velocities combined with the typical geometries, intensity scintillation is not easily observed by ground-based GNSS receivers, while fluctuations on the signal phase are more easily detected (Rino and Matthews 1980). At auroral and polar latitudes, therefore, fading does not tend to occur on ground-based GNSS receivers. However, when the phase fluctuations become very large (i.e. $\sigma_{\phi}>1 \mathrm{rad}$ ), losses of lock can be induced.
The temporal scale of ionospheric scintillation observed through ground-based GNSS receivers can be shorter than about $3 \mathrm{~s}$, with the shortest scales typically associated with high levels of scintillation. For this reason, a sampling rate of $50 \mathrm{~Hz}$ is typically used to capture the entire range of scales that contribute to scintillation (van Dierendonck et al. 1993). This is particularly useful at higher latitudes, where the relative plasma drift can be much faster (i.e. about $1 \mathrm{~km} / \mathrm{s}$ ) than at low latitudes (i.e. about $200 \mathrm{~m} / \mathrm{s}$ ). In these cases, a sampling rate of $1 \mathrm{~Hz}$ is not enough to capture the contribution from all of the scales through which scattering takes place that can originate scintillation.

Like ground-based receivers, spaceborne receivers can experience a reduction in the accuracy of the carrier phase observables due to ionospheric scintillation, as well as losses of lock if scintillation is strong. However, for spaceborne receivers, the typical temporal scales for scintillation-induced signal perturbations are much shorter than in the case of ground-based receivers. This can be seen by using the concept of Fresnel frequency under the weak scattering approximation. In this case, the Fresnel frequency is given by 

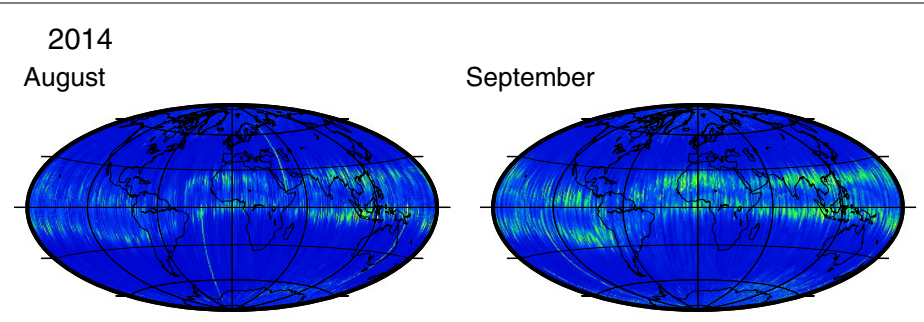

\section{October}

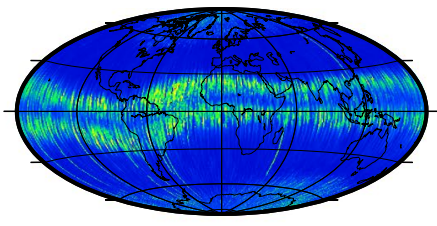

2015

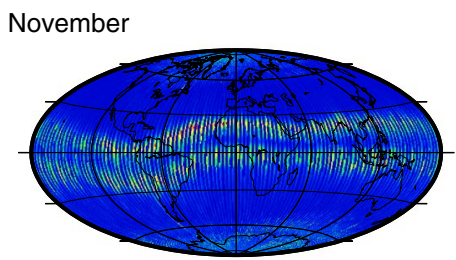

December

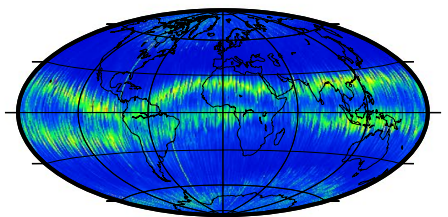

January

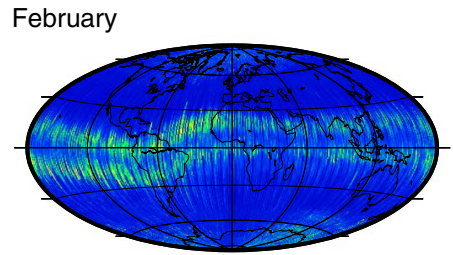

March
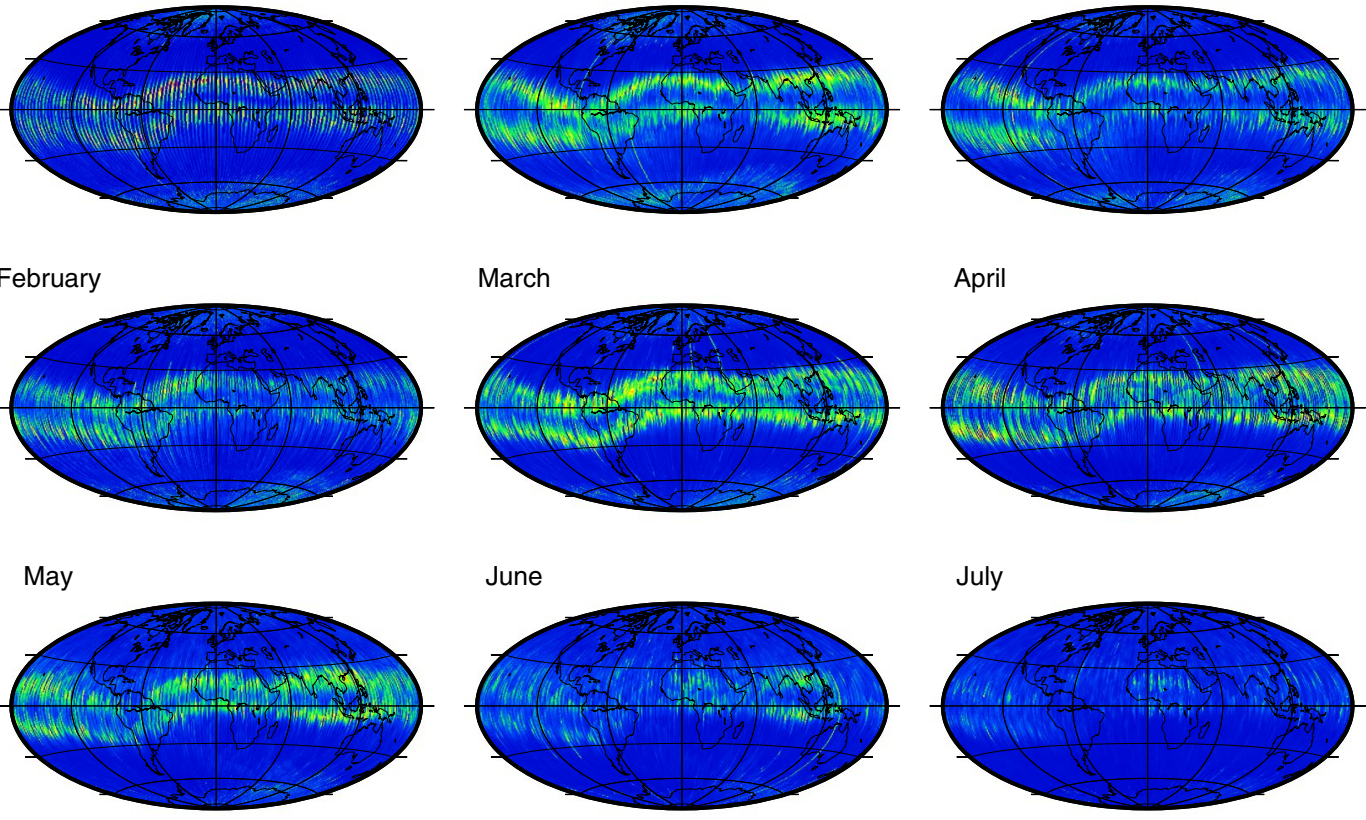

June
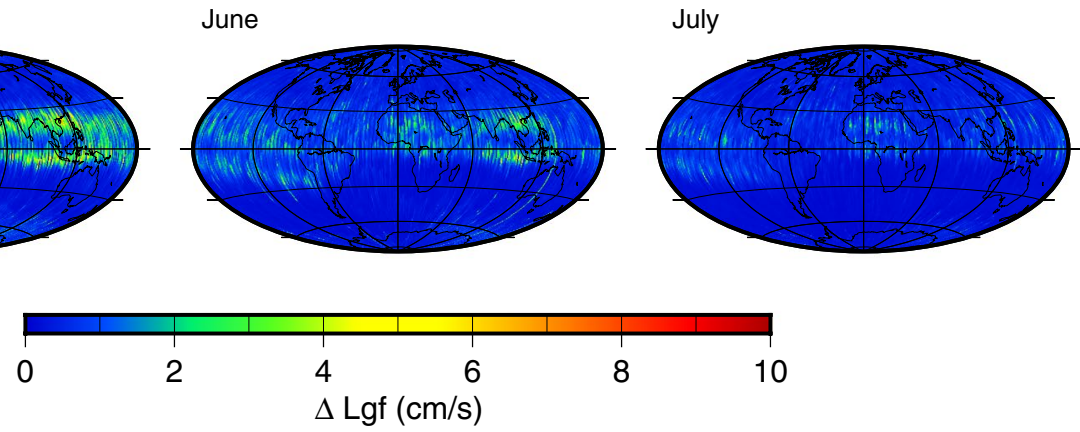

Fig. 7 Monthly maps of ionospheric variability detected by Swarm-A using time derivatives of the geometry-free combination of dual-frequency carrier phase observations accumulated over the respective month and averaged over $1^{\circ} \times 1^{\circ}$ bins

$$
v_{\mathrm{F}}=\frac{V^{\mathrm{REL}}}{d_{\mathrm{F}}}
$$

where $d_{\mathrm{F}}$ is the Fresnel scale of the problem and $V^{\mathrm{REL}}$ is the relative drift between the ray path velocity and the plasma drift velocity. In the case of spaceborne receivers, $V^{\mathrm{REL}}$ is larger than for ground-based observations and therefore very high sampling rates (e.g. $50 \mathrm{~Hz}$ ) are needed to capture the scintillation-induced signal perturbations. For GPS receivers on low flying satellites like Swarm, the signal processing in the receiver indeed happens at high frequencies (e.g. analogue-to-digital conversion, acquisition, tracking), which means that the intrinsic signal processing is exposed to the appropriate time scales of the scintillation-induced signal. However, the observations are generally delivered with a lower sampling rate (e.g.
0.1 or $1 \mathrm{~Hz}$ ), which means that the observations can show only a subset of the scintillation-induced signal perturbations, in this case only from larger scales.

Furthermore, as the plasma structures containing smaller-scale irregularities capable of generating scintillation at L band are field-line aligned, spaceborne receivers can be more or less exposed to scintillation, depending on the relative orientation between a given raypath and magnetic field lines. In addition, the scintillation-induced signal perturbations also depend on the spacecraft altitude. In Zangerl et al. (2014) and Buchert et al. (2015), it is observed that the Swarm satellites could be travelling within in situ plasma gradients. In case the Swarm GPS receivers are flying within a plasma bubble or plumes of ionisation, fluctuations on the phase can be detected without any substantial deterioration of the 
signal-to-noise ratio. Because the receiver is in the near field (i.e. within the Fresnel scale of the problem in the weak scattering approximation), destructive interference would not be fully developed to cause fading, but the propagation through gradients in the refractive index distribution would be enough to superimpose a signature on the carrier phase.

The POD of the Swarm satellites is based on the ionosphere-free combination of GPS code and carrier phase observations. This linear combination of dual-frequency observables eliminates the first-order ionospheric error. The error induced by ionospheric scintillation is, however, not directly and easily scalable according to the carrier frequency and, hence, does not necessarily cancel out in this combination. Higher-order ionospheric terms, which are not modelled in the Swarm POD, could also introduce errors. However, the residual errors of the Swarm satellites show a remarkable concentration around the peaks of the equatorial anomaly and at auroral and polar latitudes (see also Figs. 9, 12). Higher-order terms do depend not only on the carrier frequency, but also on the relative orientation to the magnetic field (Budden 1988; Davies 1990; Hoque and Jakowski 2006). In case of Swarm, the orientation to the magnetic field is completely different at higher latitudes, where the field lines are nearly vertical, than at lower latitudes, where the field lines are nearly horizontal. Furthermore, the multipath-induced error is expected to be small, assuming that for the onboard GPS receivers the local multipath was minimised during the design phase, and remaining multipath errors are largely taken into account using an inflight PCV map calibration. Consequently, the scintillation-induced error is the most likely candidate to explain the systematic residual errors that are observed for the Swarm POD.

The ionospheric scintillation-induced error depends on the transfer function of the particular architecture of the receiver utilised. Hence, different receivers may respond differently to the same ionospheric scintillation event, particularly in the strong scintillation regime where nonlinearity appears. This explains why various satellite missions at similar altitudes can observe different scintillation-induced effects. The receiver-dependent response allows tuning of the receivers parameters, in order to maximise the tracking in the presence of ionospheric scintillation, similarly to what is done in groundbased GPS scintillation monitors (van Dierendonck et al. 1993). In the particular case of Swarm, the tracking bandwidths have been widened in an attempt to increase the robustness against scintillation. The widening of the tracking bandwidth on L2 or L1, or both, can improve the capability of tracking in the presence of a reduced signal-to-noise, as well as of larger phase variations (van
Dierendonck 1996). However, it would also produce noisier observations of the carrier phases. To obtain optimal receiver settings with respect to these two effects, a trade-off has to be made.

\section{Swarm GPS receiver tracking loop modifications}

On 6 May 2015, the tracking loops of the GPS receiver on Swarm-C were modified in order to make the receiver more robust against ionospheric scintillation. The bandwidth of the L1 carrier loop was increased by $50 \%$ from 10 to $15 \mathrm{~Hz}$, while the L2 carrier loop bandwidth was increased by $100 \%$ from 0.25 to $0.5 \mathrm{~Hz}$. The bandwidth of the $\mathrm{P}(\mathrm{Y})$ code loop was also increased by $100 \%$, even though this is not of immediate relevance in the present context. In addition, the fast reacquisition of the L1-carrier tracking was enabled by increasing the retry counter from zero to five. This was done to prevent the GPS receiver from immediately trying to acquire a new GPS satellite after losing lock during scintillation conditions. Finally, the number of tracked satellites required by the onboard Kalman filter for calculating a valid navigation solution was reduced from three to one, in order to try to maintain a clock solution at times with poor GPS tracking during severe scintillation conditions. Because these modifications were first implemented on Swarm$\mathrm{C}$ only, their impact can again be assessed by comparing the performance of this satellite with Swarm-A. On the same day the new tracking loops were implemented on Swarm-C, the FoV settings on Swarm-A and Swarm-B were also updated to $88^{\circ}$, which means that, except for the updated tracking loops, the GPS receivers on Swarm$A$ and Swarm-C have identical settings.

\section{Impact on collected GPS data}

Figure 1 shows that the implemented tracking loop updates have a positive impact on the amount of collected GPS observations. The average number of tracked GPS satellites per epoch increases from around 7.6 to more than 7.7, which is an increase of around $1.3 \%$. Due to the improved robustness of the tracking loops, the GPS receiver starts collecting observations at slightly lower elevations, as shown in Fig. 2. Figure 1 shows occasional dips in the number of tracked GPS satellites that are not related to GPS receiver modifications. There is a small dip around February 2014 and even larger dips around October-November 2014 and March 2015. At these times, the Swarm GPS receivers are affected by equatorial scintillation, which lead to occasional tracking losses. This was also shown by Buchert et al. (2015), who analysed the Swarm GPS tracking losses in February 2014.

As explained in the previous section, equatorial scintillation is most intense in the hours after sunset. Around 
February and October-November 2014, the Swarm descending equator crossings are during these post-sunset hours, while around March 2015, the ascending equator crossings experience the post-sunset local time (van den IJssel et al. 2015). Around June 2014 and July 2015, the equator crossings are also during the post-sunset hours. However, during summer there is minimum ionospheric scintillation and this explains the absence of a dip in GPS tracking during these periods. For Swarm-B, the two larger dips are slightly delayed compared with the two other Swarm satellites, which can be explained by the slow drift between the orbital planes of the high flying Swarm-B satellite and the lower pair, which leads to a growing difference in local time. Because of its higher altitude, Swarm-B is also less affected by scintillation than the lower pair, and therefore the dips in GPS tracking are smaller for this satellite. Around December 2015, the ascending equator crossings of the lower satellite pair are again during the post-sunset hours. However, Fig. 1 shows no dip in the GPS tracking of these satellites around this time, which confirms the improved robustness of the updated tracking loops.

\section{Impact on reduced-dynamic POD performance}

The impact of the updated tracking loops on the reduceddynamic POD performance is assessed by comparing the performance of Swarm-A and Swarm-C for the time period between 7 May and 21 July 2015. During this period, the GPS receivers of these satellites have identical settings, except for the updated tracking loops that are implemented on Swarm-C only. Widening the carrier loop bandwidth increases the thermal noise of the carrier phase observations, especially at low elevation angles. However, the left panel of Fig. 8 shows that the daily rms error of the reduced-dynamic GPS carrier phase residuals is significantly lower for Swarm-C compared with
Swarm-A. This suggests that the overall error budget of the carrier phase observations was dominated by scintillation-induced errors, which exceeded the contribution of thermal noise in the carrier phase observations. The thermal noise increase caused by widening the tracking loops did not counteract the benefit obtained from the reduction in those systematic errors.

Similar features are visible for the daily rms errors of both satellites, due to the small distance between the two satellites, except for the outlier on 25 June 2015, which is only visible for Swarm-A. On this day, an orbit manoeuvre was executed for Swarm-A. Because accurate manoeuvre information is not readily availably and the number of orbit manoeuvres is limited, such orbit manoeuvres are currently not properly taken into account in the reduced-dynamic POD, and this leads to higher carrier phase residuals around manoeuvres. For Swarm-A, the carrier phase residual errors shown in Fig. 8 are significantly lower compared with the errors shown in Figs. 5 and 6, even though the tracking loops of this satellite were not modified and a larger GPS antenna elevation mask was used. This can be explained by the minimum ionospheric scintillation during summer, which leads to lower carrier phase residuals.

The expected reduction in systematic errors in the carrier phase observations of Swarm-C is confirmed by Fig. 9. This figure shows polar plots of the distribution of the rms error of the reduced-dynamic carrier phase residuals accumulated over the selected time period and averaged over $1^{\circ} \times 1^{\circ}$ bins. The top panel shows that for Swarm-A the rms error is less than $5 \mathrm{~mm}$ for most of the Earth, but there are systematically larger errors close to the geomagnetic poles and, to a lesser extent, also along the geomagnetic equator. The bottom panel of Fig. 9 shows that for Swarm-C, these systematic errors are significantly reduced, due to the improved robustness of the
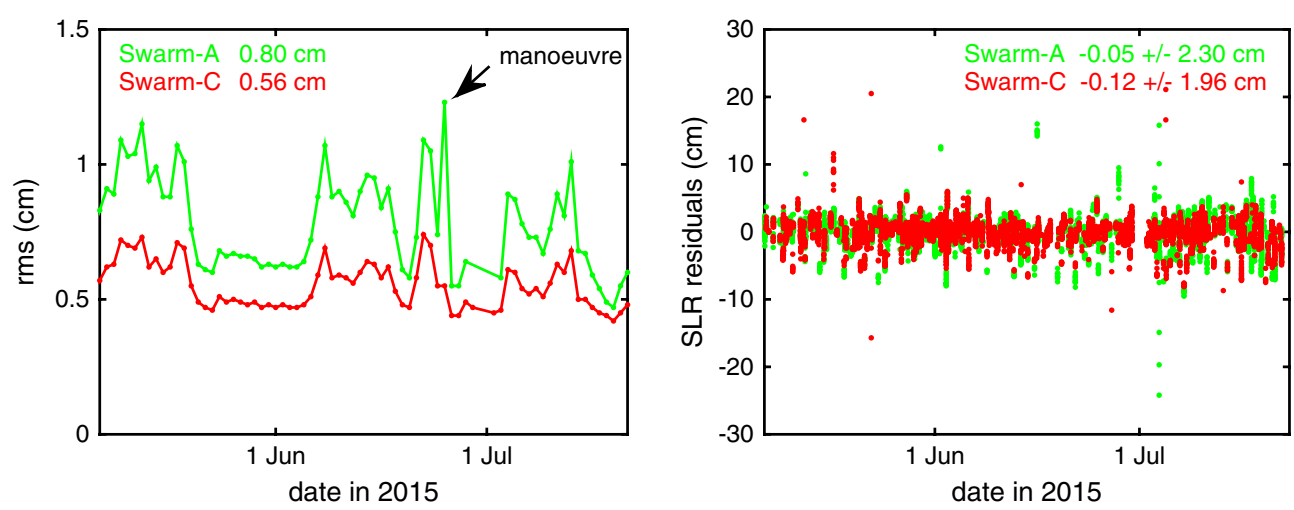

Fig. 8 Daily rms errors of the ionosphere-free carrier phase residuals (left panel) and SLR residuals (right panel) for the reduced-dynamic orbits of Swarm-A and Swarm-C 


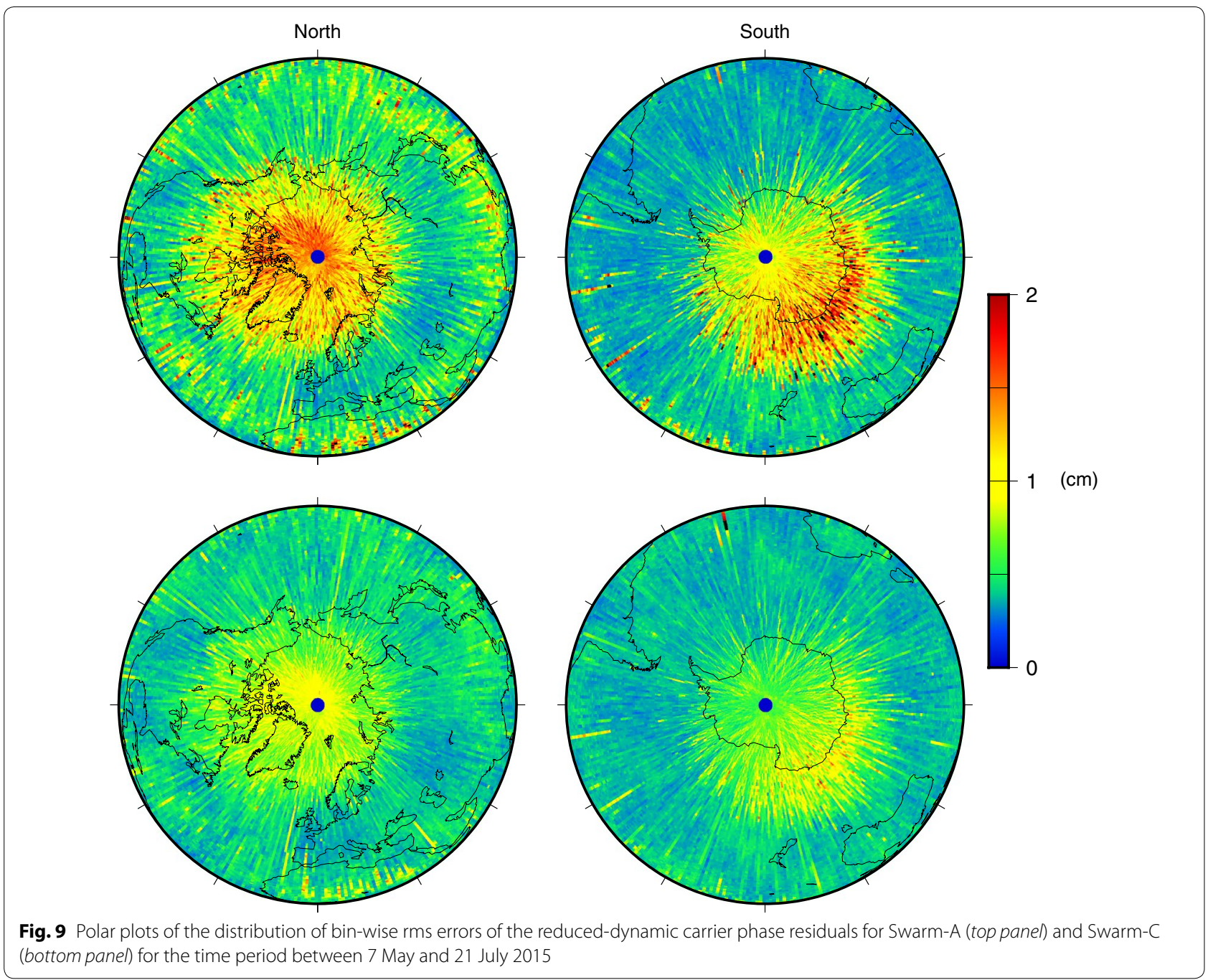

new tracking loops. In addition, no significant degradation is visible at the midlatitudes, which suggests that the widening of the tracking loop bandwidths has no negative impact on carrier phase observations that are not affected by ionospheric scintillation. The SLR validation shown in the right panel of Fig. 8 confirms the positive impact of the updated tracking loops on the reduceddynamic POD performance. For the selected time period, the standard deviation of the Swarm-C SLR residuals is better than $2 \mathrm{~cm}$, compared with about $2.3 \mathrm{~cm}$ for Swarm-A.

\section{Impact on kinematic POD performance}

The impact of the updated tracking loops on the kinematic POD performance is assessed by a similar comparison between Swarm-A and Swarm-C. The kinematic POD technique relies completely on the GPS observations, and therefore it is not possible to obtain a solution during periods with few or no observations. Figure 10 shows that for the selected period, the kinematic orbits of Swarm-C are more robust, with on average position solutions for $99.6 \%$ of the GPS observations epochs, compared with $98.9 \%$ position solutions for Swarm-A. On 25 June 2015, both satellites have slightly less available positions solutions, which is due to missing clock observations in the CODE 5-s clock product of this day.

The left panel of Fig. 11 shows that also for kinematic POD, the modified tracking loops lead to lower carrier phase residuals. The kinematic carrier phase residuals of Swarm-A are about $5.6 \mathrm{~mm}$, while the Swarm-C residuals are at the $4 \mathrm{~mm}$ level. The SLR validation shown in the right panel of Fig. 11 confirms the positive impact of the updated tracking loops on the kinematic POD performance. For Swarm-C, the standard deviation of the SLR residuals for the selected period is about $2.7 \mathrm{~cm}$, compared with more than $3 \mathrm{~cm}$ for Swarm-A.

The quality of the kinematic orbits can also be assessed by comparing them to the reduced-dynamic orbits. 


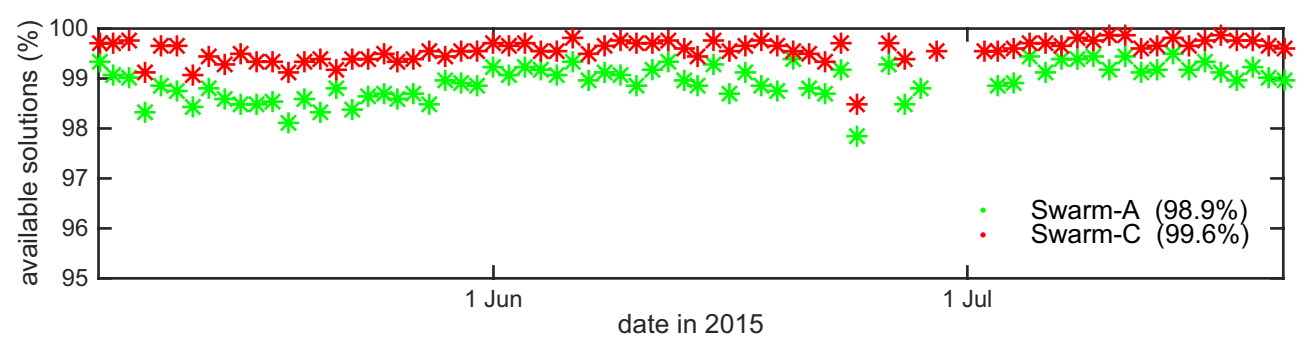

Fig. 10 Overview of available position solutions per kinematic PSO for Swarm-A and Swarm-C
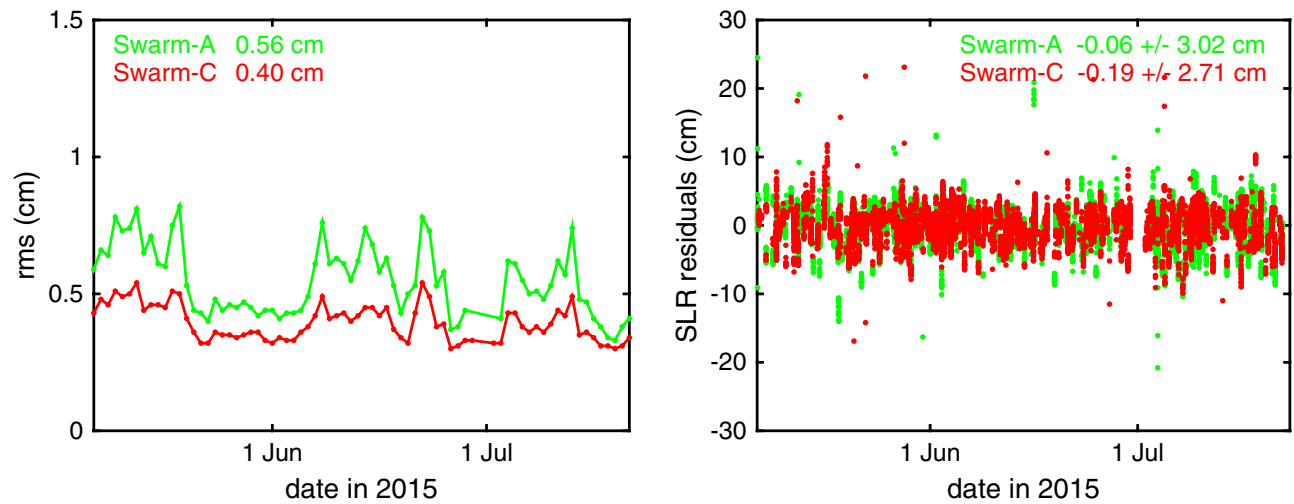

Fig. 11 Daily rms errors of the ionosphere-free carrier phase residuals (left panel) and SLR residuals (right panel) for the kinematic orbits of Swarm-A and Swarm-C

Figure 12 shows polar plots of the rms of the 3D differences between these orbits accumulated over the selected time period and averaged over $1^{\circ} \times 1^{\circ}$ bins. The top panel shows that the orbit differences for Swarm-A are significantly larger close to the geomagnetic poles and, to a lesser extent, also along the geomagnetic equator. The stripes that are visible in this figure are caused by several poorly determined orbit tracks. As mentioned before, kinematic orbits are more sensitive to a degraded GPS tracking performance than reduced-dynamic orbits, and therefore the larger orbit differences at these locations indicate the reduced quality of the kinematic orbits. The bottom panel of Fig. 12 shows that these large orbit differences are greatly reduced for Swarm-C, which again confirms the positive impact of the modified tracking loops on the quality of the kinematic orbit solutions.

It is expected that the modified tracking loops will also improve the determination of kinematic baselines, which suffers from the degraded Swarm GPS receiver performance over the geomagnetic poles. With more robust tracking loops, the larger errors over these areas are greatly reduced, as shown in Figs. 9 and 12. In addition, it is anticipated that the modified tracking loops will also reduce the systematic errors along the geomagnetic equator that are present in the gravity fields determined from Swarm GPS data. These systematic gravity field errors vary in time and are larger when the satellite crosses the equator during the post-sunset hours. They also show a seasonal dependency, with larger errors during equinoxes and minimum errors during summer. This suggests that the errors are caused by equatorial scintillation. Jäggi et al. (2015) show that these systematic gravity field errors can be reduced by discarding GPS data that are affected by large ionospheric changes. This procedure was also applied for the GOCE satellite, which showed similar disturbances (Jäggi et al. 2015). However, the Swarm receiver was found to be even more sensitive to this effect than the GOCE receiver, and lower threshold values had to be used for the rejection of Swarm GPS data. It is expected that with more robust tracking loops, the threshold value for the Swarm GPS data can also be relaxed.

\section{Conclusions}

The Swarm state-of-the-art GPS receivers deliver highquality data with an almost continuous data coverage, which allows precise orbit determination with a few $\mathrm{cm}$ accuracy. Despite this good performance, the onboard receiver settings have been modified several times during 


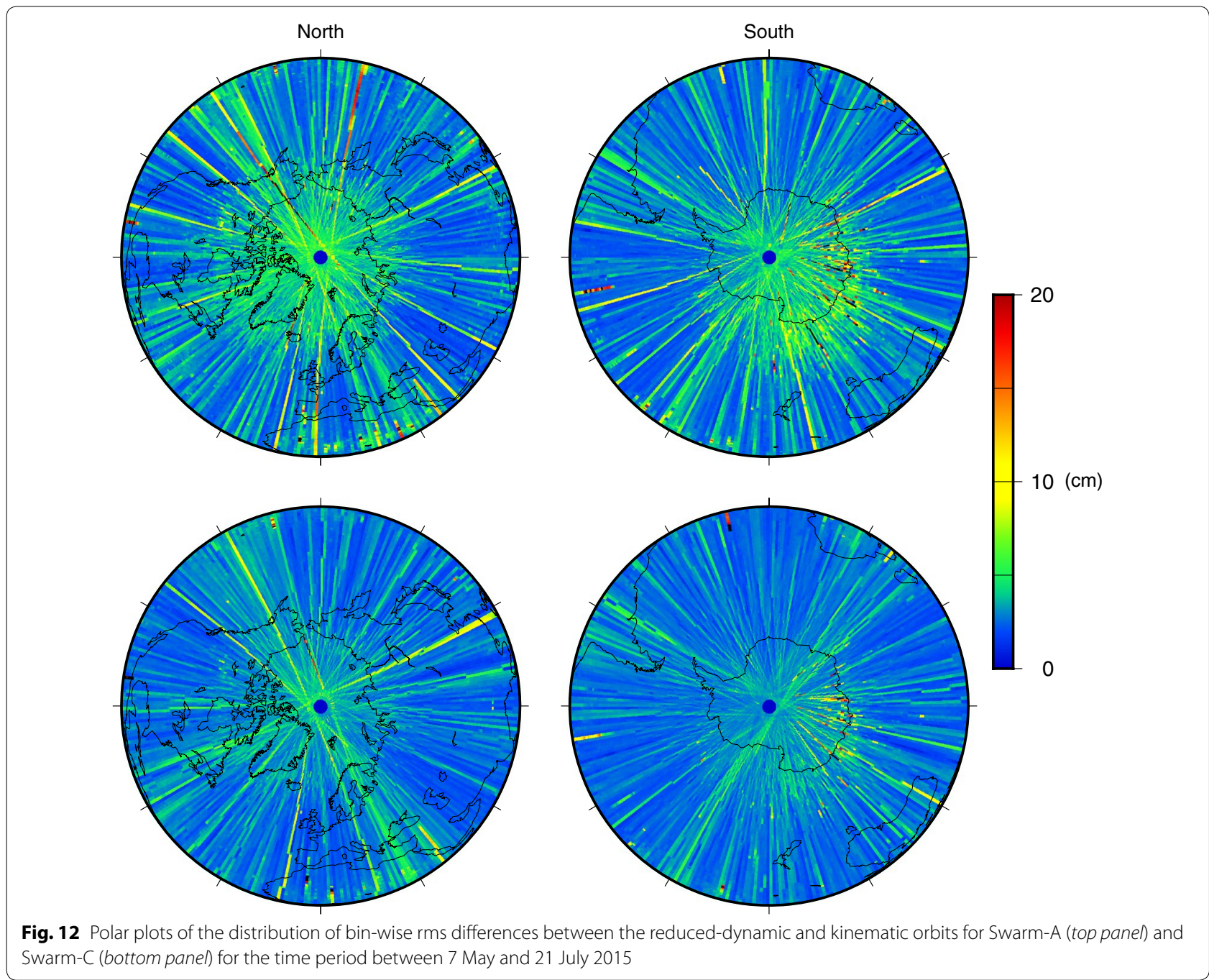

the mission to further improve the Swarm GPS tracking performance. With only eight channels available for dual-frequency tracking, the number of tracked satellites is relatively low for the Swarm GPS receivers. The GPS antenna elevation mask has therefore slowly been reduced from $10^{\circ}$ to $2^{\circ}$, and this increases the amount of collected GPS observations by more than $3 \%$. The additional observations at low elevations have a higher noise level and increase the forward-backward asymmetry of the observations in the antenna frame. Nonetheless, independent SLR validation shows that the additional observations have a positive impact on both the reduceddynamic and kinematic orbit accuracy.

In addition, the Swarm GPS receivers are affected by ionospheric scintillation, which slightly degrades their performance when flying close to the geomagnetic poles and the geomagnetic equator. This affects in particular the quality of the kinematic orbits, which show larger errors at these locations. To improve the robustness of the Swarm
GPS receivers for ionospheric scintillation, the receiver settings have been modified by widening the bandwidths of the tracking loops. With the updated tracking loops, the amount of collected GPS observations increases by about $1.3 \%$ and occasional tracking losses due to severe equatorial ionospheric scintillation are greatly reduced. The wider bandwidths of the carrier loops also significantly reduce the large carrier phase residuals close to the geomagnetic poles, and no degradation is visible for GPS observations at midlatitudes. Both the reduced-dynamic and kinematic orbits show lower SLR residuals, which indicates that the updated tracking loops also improve the POD performance for both orbit types.

The accuracy of the Swarm PSOs was already sufficient to meet the geotagging requirements for the magnetic and electric field observations, and it is therefore not expected that the improved orbit accuracy due to the receiver modifications will benefit the Swarm geomagnetic and electric field results. During 
times of severe scintillation, however, the geomagnetic and electric field observations benefit from the more robust GPS receiver clock solution. Because the determination of thermospheric neutral densities relies heavily on the quality of the GPS tracking data, it is likely that the improved GPS receiver performance will lead to more accurate densities. The largest impact of the GPS receiver modifications, however, is expected for the Swarm gravity field recovery results. This recovery will benefit from the improved quality of the kinematic orbits due to the GPS receiver modifications. In addition, it is expected that the more robust tracking loops will improve the kinematic baseline determination. Such improved kinematic baselines could also contribute to the Swarm gravity field recovery. The anticipated reduction in the systematic gravity field errors along the geomagnetic equator due to the modified tracking loops could lead to another potential improvement in gravity field recovery.

Although the modified tracking loops significantly improve the Swarm GPS performance over areas affected by ionospheric scintillation, noticeably larger errors at these locations remain present in Figs. 9 and 12 . These results are obtained during the summer period, which generally has low ionospheric scintillation. It is expected that during more severe ionospheric scintillation conditions the remaining errors will be even larger. It is therefore recommended to widen the L2 carrier loop bandwidth by e.g. another $50 \%$, to further improve the robustness of the Swarm GPS receivers. By implementing this update first on Swarm-C, it is again possible to assess the impact using a comparison with Swarm-A. Other LEO satellites with GPS receivers that encounter similar disturbances, like e.g. Sentinel-1A, might also benefit from this analysis.

\section{Authors' contributions}

JIJ performed the study and drafted the manuscript. BF provided the section about ionospheric scintillation. OM contributed to the interpretation of the results. All authors read and approved the final manuscript.

\section{Author details \\ ${ }^{1}$ Faculty of Aerospace Engineering, Delft University of Technology, Delft, The Netherlands. ${ }^{2}$ Department of Electronic and Electrical Engineering, University of Bath, Bath, UK. ${ }^{3}$ Deutsches Zentrum für Luft- und Raumfahrt, German Space Operations Center, Oberpfaffenhofen, Germany.}

\section{Acknowledgement}

The authors acknowledge ESA for the provision of the Swarm data. Parts of the work described in this study were financed through ESA Contract No. 4000102140/10/NL/JA. The helpful information provided by Christian Siemes about all Swarm updates is very much appreciated. Two anonymous reviewers are thanked for their comments.

\section{Competing interests}

The authors declare that they have no competing interests.

Received: 4 January 2016 Accepted: 3 May 2016

Published online: 23 May 2016

\section{References}

Aarons J (1982) Global morphology of ionospheric scintillations. Proc IEEE 70(4):360-378

Allende-Alba G, Montenbruck O (2015) Robust and precise baseline determination of distributed spacecraft in LEO. Adv Space Res. doi:10.1016/j. asr.2015.09.034

Basu S, MacKenzie E, Basu Su (1988) lonospheric constraints on VHF/UHF communications links during solar maximum and minimum periods. Radio Sci 23(3):363-378

Bock H, Dach R, Jäggi A, Beutler G (2009) High-rate GPS clock corrections from CODE: support of $1 \mathrm{~Hz}$ applications. J Geod 83(11):1083-1094. doi:10.1007/s00190-009-0236-1

Briggs BH, Parkin IA (1963) Observations of radio star scintillations and spreadF echoes over a solar cycle. J Atmos Terr Phys 26:1-23

Buchert S, Zangerl F, Sust M, André M, Eriksson A, Wahlund J-E, Opgenoorth $H$ (2015) SWARM observations of equatorial electron densities and topside GPS track losses. Geophys Res Lett 42:2088-2092. doi:10.1002/2 015GL063121

Budden KG (1988) The propagation of radio waves: the theory of radio waves of low power in the ionosphere and magnetosphere. Cambridge University Press, Cambridge

Davies K (1990) Ionospheric radio. In: IEE electromagnetic waves series 31, IET

ESA (2015) Swarm GPSR update. https://earth.esa.int/web/guest/missions/ esa-operational-eo-missions/swarm/news. Accessed 1 July 2015

Flechtner F, Morton P, Watkins M, Webb F (2014) Status of the GRACE follow-on mission. In: Marti U (ed) Gravity, geoid and height systems, international association of geodesy symposia 141. Springer, Berlin, pp 117-121

Friis-Christensen E, Lühr H, Knudsen D, Haagmans R (2008) Swarm—an earth observation mission investigating geospace. Adv Space Res 41 (1):210216. doi:10.1016/j.asr.2006.10.008

Hoque M, Jakowski N (2006) Higher order ionospheric effects in precise GNSS positioning. J Geod 81(4):259-268. doi:10.1007/s00190-006-0106-0

Hoque M, Jakowski N, Gerzen T, Berdermann J (2014) A new approach for receiver bias estimation and TEC calibration for SWARM-GPS paths. Poster presented at the 3rd Swarm science meeting, Copenhagen, Denmark, 19-20 June 2014

Jäggi A, Dach R, Montenbruck O, Hugentobler U, Boch H, Beutler G (2009) Phase center modeling for LEO GPS receiver antennas and its impact on precise orbit determination. J Geod 83(12):1145-1162. doi:10.1007/ s00190-009-0333-2

Jäggi A, Bock H, Meyer U, Beutler G, van den IJssel J (2015a) GOCE: assessment of GPS-only gravity field determination. J Geod 89(1):33-48. doi:10.1007/ s00190-014-0759-z

Jäggi A, Dahle C, Arnold D, Bock H, Meyer U, Beutler G, van den IJssel J, (2015) Swarm kinematic orbits and gravity fields from 18 months of GPS data. Adv Space Res. doi:10.1016/j.asr.2015.10.035

Kintner PM, Ledvina BM, de Paula ER (2007) GPS and ionospheric scintillations. Space Weather 5(9):S09003. doi:10.1029/2006SW000260

Kramer L, Goodman JL (2005) lonospheric instability observed in low Earth orbit using global positioning system. J Spacecr Rockets 42(1):124-131

Montenbruck O, Garcia-Fernandez M, Williams J (2006) Performance comparison of semi-codeless GPS receivers for LEO satellites. GPS Solut 10:249-261. doi:10.1007/s10291-006-0025-9

Olsen N, Friis-Christensen F, Floberghagen R, Alken P, Beggan C, Chulliat A, Doornbos E, Encarnação J, Hamilton B, Hulot G, van den IJssel J, Kuvshinov A, Lesur V, Lühr H, Macmillan S, Maus S, Noja M, Olsen P, Park J, Plank G, Püthe Ch, Rauberg J, Ritter P, Rother M, Sabaka T, Schachtschneider R, Sirol O, Stolle C, Thébault E, Thomson A, Tøffner-Clausen L, Velímský J, Vigneron P, Visser P (2013) The Swarm satellite constellation application and research facility (SCARF) and Swarm data products. Earth Planets Space 65(11):1189-1200. doi:10.5047/eps.2013.07.001

Pearlman MR, Degnan JJ, Bosworth JM (2002) The international laser ranging service. Adv Space Res 30(2):135-143. doi:10.1016/ S0273-1177(02)00277-6

Rino CJ, Matthews SJ (1980) On the morphology of auroral zone radio wave scintillation. J Geophys Res Space Phys 85(A8):4139-4151

Siemes C, Encarnação J, Doornbos E, van den IJssel J, Kraus J, Pereštý R, Grunwaldt L, Apelbaum G, Flury J, Olsen P (2015) Swarm accelerometer data processing from raw accelerations to thermospheric neutral 
densities. Earth Planets Space (Swarm special issue). doi:10.1186/ s40623-016-0474-5

Skone S, Knudsen K, de Jong M (2001) Limitations in GPS receiver tracking performance under ionospheric scintillation conditions. Phys Chem Earth A 26:613-621. doi:10.1016/S1464-1895(01)00110-7

van den IJssel J, Visser P, Doornbos E, Meyer U, Bock H, Jäggi A (2011) GOCE SSTI L2 losses and their impact on POD performance. In: Proceedings of the 4th international GOCE user workshop, Munich, Germany, ESA SP-696, pp 1-6

van den IJssel J, Encarnação J, Doornbos E, Visser P (2015) Precise science orbits for the Swarm satellite constellation. Adv Space Res 56(6):10421055. doi:10.1016/j.asr.2015.06.002

van Dierendonck AJ, Klobuchar J, Hua Q (1993) Ionospheric scintillation monitoring using commercial single frequency C/A code receivers. In: Proceedings of the 6 th international technical meeting of the satellite division of the institute of navigation (ION GPS-93), Arlington, VA, pp 1333-1342 van Dierendonck AJ (1996) GPS receivers. In: Parkinson BW, Spilker JJ (eds) Global positioning system: theory and applications, vol 1. AIAA, Washington, pp 329-407. doi:10.2514/4.866388

Visser P, Doornbos E, van den IJssel J, Encarnação J (2013) Thermospheric density and wind retrieval from Swarm observations. Earth Planets Space 65(11):1319-1331. doi:10.5047/eps.2013.08.003

Wermuth M, Montenbruck O, van Helleputte T (2010) GPS high precision orbit determination software tools (GHOST). In: 4th international conference on astrodynamics tools and techniques, Madrid, Spain, 3-6 May 2010

Woo KT (2000) Optimum semicodeless carrier-phase tracking of L2. Navigation 47(2):82-99

Zangerl F, Griesauer F, Sust M, Montenbruck O, Buchert B, Garcia A (2014) SWARM GPS precise orbit determination receiver initial in-orbit performance evaluation. In: Proceedings of the 27 th international technical meeting of the satellite division of the institute of navigation (ION GNSS+), Tampa, Florida, 2014, pp 1459-1468

\section{Submit your manuscript to a SpringerOpen ${ }^{\circ}$ journal and benefit from:}

- Convenient online submission

- Rigorous peer review

- Immediate publication on acceptance

- Open access: articles freely available online

- High visibility within the field

- Retaining the copyright to your article 